\title{
Consequences of Simulated Microgravity in Neural Stem Cells: Biological Effects and Metabolic Response
}

Marianna Silvano ${ }^{1, \#}$, Evelina Miele ${ }^{1,2, \#, ~ M a r i a c r i s t i n a ~ V a l e r i o ~}{ }^{3, \#}$, Luca Casadei $^{3}$, Federica Begalli' ${ }^{1}$, Antonio Francesco Campese ${ }^{1}$, Zein Mersini Besharat ${ }^{1}$, Vincenzo Alfano ${ }^{1}$, Luana Abballe ${ }^{1}$, Giuseppina Catanzaro ${ }^{4}$, Maddalena Napolitano $^{1}$, Alessandra Vacca $^{4}$, Isabella Screpanti ${ }^{1,2}$, Cesare Manetti ${ }^{3}$, Elisabetta Ferretti ${ }^{4 * \$}$ and Agnese Po $^{1 \$}$

${ }^{1}$ Department of Molecular Medicine, Sapienza University, Rome 00161, Italy

${ }^{2}$ Center for Life Nano Science, Sapienza, Istituto Italiano di Tecnologia, Rome 00161, Italy

${ }^{3}$ Department of Chemistry, Sapienza University, 00185 Rome, Italy

${ }^{4}$ Department of Experimental Medicine, Sapienza University, Rome 00161, Italy

\#These authors contributed equally

$\$$ co-senior authors

\section{Abstract}

Objective: Microgravity was often shown to cause cell damage and impair cell cycle in a variety of biological systems. Since the effects on the neural system were poorly investigated, we aimed to gain insight into how biological processes such as cell cycle, cell damage, stemness features and metabolic status are involved in neural stem cells (NSC) when they experience simulated microgravity. We also wished to investigate whether these modulations were transient or permanent once cells were returned to normal gravity.

Methods: NSC were isolated from mouse cerebella and cultured in the Rotary Cell Culture System (RCCS) to model microgravity. We analyzed cell cycle, stress and apoptotic response. We also performed a $1 \mathrm{H}$ NMR-based metabolomic analysis and evaluation of stemness features of NSC in simulated microgravity and once in the returned to normogravity cell culture.

Results: Biological processes and metabolic status were modulated by simulated microgravity. Cells were arrested in S-phase together with enhanced apoptosis. Metabolic changes occurred in NSC after simulated microgravity. Interestingly, these modulations were transient. Indeed, stemness features and metabolic footprint returned to basal levels after few days of culture in normal conditions. Moreover NSC clonogenic ability was not impaired.

Conclusions: Our data suggest that simulated microgravity impacts on NSC biological processes, including cell cycle and apoptosis. However, NSC does not suffer from permanent damage.

Keywords: Neural stem cells; Simulated microgravity; Metabolic profile; Cell cycle; Apoptosis

\section{Introduction}

The opportunity of spaceflights and the prolonged journey on nearearth-orbit space stations raised questions about the effects of reduced gravity on physiological and pathological processes.

Several physiopathological effects have been linked to the orbital flight and it has been established that the longer the stay, the more likely that astronauts will encounter some acute and chronic illnesses [1].

More evident negative effects from the absence of regular gravity reside in muscle and skeletal systems (muscular atrophy and osteopenia) [2,3], and at the hematological level, with changes in erythrocyte morphology and impaired immunoresponse [4]. Nonetheless, microgravity studies underlined a close relationship between mechanical load and biological functions, showing induction of adaptive responses at the molecular, cellular and systemic levels. This adaptive response occurs via changes of specific functions, such as cell cycle and stress response [4-8], and involves activation of metabolic pathways and of specific gene expression programs $[9,10]$. Unfortunately, such an adaptive response is not always appropriate and efficient, thus a number of cell damages can accumulate, which are then responsible for the physiopathological conditions observed [11].

The Rotary Cell Culture System (RCCS) is a tool developed by NASA able to model microgravity. Researchers have taken advantage of the RCCS system to analyze the effects of reduced gravity on a large number of biological systems.

Cerebellar neural stem cells (NSC) are characterized by the ability to self-renew in vitro and by pluripontency, generating the main phenotype of the nervous system. We have previously shown that the embryonic stemness molecule Nanog is an important transcription factor in this context [12] and governs self-renewal and cell cycle [13].

Metabolomics takes advantage of the accessibility of highthroughput technologies, i.e. NMR, to investigate perturbations caused by external stimuli to the system (cells/organism) [14]. This perturbation will be responsible for the change in the concentration of certain lowmolecular weight metabolites. These results will be then integrated in cell processes to understand metabolic changes determined by the phenomenon under study.

Here we present a deep analysis of NSC exposed to microgravity, integrating data from biological processes and metabolomic changes. Our results converge in showing that NSC are stressed by the exposure to microgravity, their cell cycle altered and apoptosis enhanced. Of note, these changes are not permanent, since the return to normal gravity restores starting features of NSC.

*Corresponding author: Elisabetta Ferretti, MD PhD, Department of Experimental Medicine - Sapienza University, Rome, Italy Viale Regina Elena, 291 - 00161 Rome, Italy, Tel: +39 0649255135; E-mail: elisabetta.ferretti@uniroma1.it

Received May 29, 2015; Accepted June 27, 2015; Published June 30, 2015

Citation: Silvano M, Miele E, Valerio MC, Casadei L, Begalli F et al. (2015) Consequences of Simulated Microgravity in Neural Stem Cells: Biological Effects and Metabolic Response. J Stem Cell Res Ther 5: 289. doi:10.4172/2157 7633.1000289

Copyright: (c) 2015 Silvano, et al. This is an open-access article distributed under the terms of the Creative Commons Attribution License, which permits unrestricted use, distribution, and reproduction in any medium, provided the original author and source are credited. 


\section{Materials and Methods}

\section{Stem cell culture}

Mouse cerebella were obtained from postnatal 4-day-old wild-type BL6 mice with the approval of Institutional Review Board [12]. Briefly, tissues were collected in HBSS supplemented with $0.5 \%$ glucose and penicillin-streptomycin, grossly triturated with serological pipette and treated with DNAse I to a final concentration of $0.04 \%$ for $20 \mathrm{~min}$. Finally, cell aggregates were mechanically dissociated using pipettes of decreasing bore size to obtain a single cell suspension. Cells were cultured as neurospheres in selective medium after centrifugation; DMEM/F12 supplemented with $0.6 \%$ glucose, $25 \mathrm{mg} / \mathrm{ml}$ insulin, 60 $\mathrm{mg} / \mathrm{ml} \mathrm{N}$-acetyl-L-cysteine, $2 \mathrm{mg} / \mathrm{ml}$ heparin, $20 \mathrm{ng} / \mathrm{ml} \mathrm{EGF} \mathrm{(Sigma}$ Aldrich), $20 \mathrm{ng} / \mathrm{ml}$ bFGF (Peprotech), $1 \times$ penicillin-streptomycin and B27 supplement without vitamin A. For the clonogenic assay, cells were plated at 5-10 cells per well into 96-well plates and cultured in selective medium; after 10 days neurospheres were manually counted and the number of neurosphere-forming cells was assessed.

\section{Experimental design and blinding}

NSC was cultured in simulated microgravity (SM) or in conventional cell culture support as control for normal gravity (1G). SM, considered similar to that of outer space, was produced by using RCCS - 4D Vessel Rotary Cell Culture System (Synthecon Inc.). Experiments were carried out for 72 hours for metabolic analysis and for five days for functional and gene expression analyses. Shorter time for experimental metabolic analysis was decided to find the most discriminatory metabolic profile, bypassing the plateau effect due to metabolic saturation of the culture medium. For rescue experiments cells were collected after 5 days of SM and then plated in $1 \mathrm{G}$ for the time indicated in the results section. Due to the subjective nature of manual counts, experiments were carried out by two independent investigators. Moreover at least 2 experiments per point were performed by blinded observer. We obtained no differences in the results from non-blinded and blinded experiments, therefore results were combined.

\section{Cell proliferation and cell cycle analysis}

To monitor individual cell divisions we used Cell Proliferation Dye eFluor 670 (eBioscience cod. 65-0840). It has a peak excitation of $647 \mathrm{~nm}$ and is detected by APC filter. We used $5 \times 10^{6}$ cells prepared in single-cell suspension and proceeded according to manufacturing instructions. For cell cycle analysis cells were fixed with $70 \%$ ethanol for $2 \mathrm{~h}$ at $+4^{\circ} \mathrm{C}$; cells were then washed and incubated overnight with $25 \mathrm{ug} /$ $\mathrm{ml}$ 7-AAD (Sigma Aldrich) and $40 \mathrm{mg} / \mathrm{ml}$ RNAse A (Sigma Aldrich) in PBS. Experiments were visualized by FACS on FACSCalibur using the CellQuest software (both from BD Biosciences, San Jose, CA, USA).

\section{Western blot and immunofluorescence}

Cells were lysed in Tris- $\mathrm{HCl} \mathrm{pH}$ 7.6, $50 \mathrm{mM}$, deoxycholic acid sodium salt $0.5 \%, \mathrm{NaCl} 140 \mathrm{mM}, \mathrm{NP}-40$ 1\%, EDTA $5 \mathrm{mM}, \mathrm{NaF} 100 \mathrm{mM}$, Na pyrophosphate $2 \mathrm{mM}$ and protease inhibitors. For immunostaining the following antibodies were used: anti-Cyclin A (H-432) (cod.sc751 Santa Cruz Biotechnology), anti-Cyclin B (H-433) (cod. sc-752 Santa Cruz Biotechnology), anti-Cyclin D2 (cod. 2924 Cell Signaling), anti-YH2AX (cod.2577 Cell Signaling), anti-HSP70 (H300) ( cod. sc33575 Santa Cruz Biotechnology), Anti-PARP p85 Fragment pAb (cod. G7341 Promega), anti-p21 (cod. sc-397 Santa Cruz Biotechnology) rabbit anti-Nanog (cod. REC-RCAB0002P-F Cosmo Bio), anti-Actin (I-19) (cod.sc-1616 Santa Cruz Biotechnology), anti-GAPDH (ab9484 Abcam). All antibodies were incubated overnight at $+4^{\circ} \mathrm{C}$ and HRP- conjugated secondary antisera (Santa Cruz Biotechnology) were used followed by enhanced chemiluminescence (PerkinElmer). For Nanog immunofluorescence, cells were plated on poly-L-lysine coated chamber slide, fixed with $4 \%$ paraformaldehyde, permeabilized with $0,1 \%$ Triton X-100 and anti-Nanog were incubated at dilution of 1:200 overnight at $+4^{\circ} \mathrm{C}$. For detection Alexa Fluor ${ }^{\circ} 488$ dye anti-rabbit secondary antibody (Lifetechnologies) was used. Nuclei were counterstained with the Hoechst reagent. DAPI staining was performed to determine the proportion of apoptotic cells by manually counting pyknotic nuclei.

\section{RNA isolation and real-time $\mathrm{qPCR}$}

RNA was extracted from cells by using TriReagent (Life Technologies). cDNA synthesis was performed using the High Capacity cDNA reverse transcription kit from Applied Biosystems. Quantitative reverse transcription (RT-PCR) analysis was performed on cDNAs employing the $\mathrm{ViiA}^{\mathrm{sa}} 7$ Real-Time PCR System (Life Technologies), using individual Taqman gene expression assay according to the manufacturer's instructions. All values were normalized to two endogenous controls (Hprt and $\beta 2$-microglobulin). ID for Taqman gene expression assays (Life Technologies): Mm03053893_gH (Cyclin B1), Mm00438070_m1 (Cyclin D2), Mm00432337_m1 (Cyclin A1), Mm0154539932_m1 (Hprt), Mm00437762_m1 (ß2-microglobulin).

\section{NMR metabolomic analysis}

For extracellular metabolite analysis, each medium sample $(2 \mathrm{ml})$ was lyophilized then dissolved in $700 \mu \mathrm{l}$ of $1 \mathrm{mM}$ TSP [sodium salt of 3-(trimethylsilyl) propionic-2,2,3,3-d4 acid], $10 \mathrm{mM}$ sodium azide $\mathrm{D} 2 \mathrm{O}$ phosphate buffer solution $(\mathrm{pH}=7.4)$ and finally homogenized by vortex mixing for $1 \mathrm{~min}$. After centrifugation (10 min, $10.000 \mathrm{RCF}$ at $22^{\circ} \mathrm{C}$ ), $600 \mu \mathrm{l}$ of each resulting supernatant was transferred to a $5-\mathrm{mm}$ NMR tube and used for the NMR analysis.

Medium samples were analyzed on a Bruker $500 \mathrm{MHz}$ DRX Bruker Avance spectrometer (Bruker Biospin, Rheinstetten, Germany). Twodimensional (2D) proton $(1 \mathrm{H})$ J-resolved (JRES) NMR spectra were collected using a double spin echo sequence with presaturation for water suppression and 4 transients per increment for a total of 32 increments. These were collected into $16 \mathrm{k}$ data points using spectral widths of $6 \mathrm{kHz}$ in $\mathrm{F} 2$ and $40 \mathrm{~Hz}$ in F1. There was a $3.0 \mathrm{~s}$ relaxation delay. Each FID was Fourier transformed after a multiplication with sine-bell window functions in both dimensions. JRES spectra were tilted by $45^{\circ}$, symmetrized about F 1 , referenced to $\delta \mathrm{HTSP}=0$ at $.0 \mathrm{ppm}$ and the proton -decoupled skyline projections (p-JRES) exported using Bruker's XWIN-NMR software.

For intracellular metabolite analysis, cell pellets were lyophilized and cryogenically grounded using Cryomill (Retsch GmbH Germany) before methanol/chloroform/water extraction (2/2/1.8) to extract polar and nonpolar metabolites $[15,16]$. Before NMR analysis, polar extracts were dissolved in $600 \mu \mathrm{l}$ of $\mathrm{D} 2 \mathrm{O}$, while non-polar extracts were dissolved in $600 \mu \mathrm{l}$ of $\mathrm{CDCl} 3$ containing $0.03 \%$ TMS (tetramethylsilane) / CD3OD solution $(2: 1, \mathrm{v} / \mathrm{v})$.

One-dimensional (1D) proton (1H) NMR spectra of intracellular metabolite extracts were collected on $700 \mathrm{MHz}$ DRX Bruker Avance spectrometer, with a TCI cryoprobe (Bruker Biospin, Rheinstetten, Germany). 1D spectra of hydrophilic extracts were acquired using presaturation for suppression of water resonance, while for lipophilic extracts a simple $90^{\circ}$ pulse-acquire sequence was used. The spectra were acquired with a relaxation delay of $5 \mathrm{~s}$ into $16 \mathrm{k}$ data points using a spectral width of $8 \mathrm{kHz}$ and 128 transients. The spectra were Fourier Transformed using an exponential window with a line broadening 
value of $0.1 \mathrm{~Hz}$, phased, baseline corrected and referenced to lactic $\delta \mathrm{H}$ acid $=1 . \delta \mathrm{H} 33$ at $=$ ppm 0.0 or ppmTMS for at polar and nonpolar fractions, respectively.

The spectra of each dataset were reduced into spectral bins with widths ranging from 0.01 to $0.02 \mathrm{ppm}$ by using the ACD intelligent bucketing method (1D NMR Manager software (ACD/Labs, Toronto, Canada), following exclusion of bins corresponding to noise and solvent peaks (residual water, $\delta \mathrm{H} 4.76-4.82 \mathrm{ppm}$; $\mathrm{CDCl} 3, \delta \mathrm{H}$ 7.45-7.50 ppm; CD3OD, $\delta \mathrm{H} 3.33-3.37$ ppm; TSP and TMS, $\delta \mathrm{H}-0.5-0.5 \mathrm{ppm})$. In order to extract useful information from these data, the binned spectra were normalized by applying the Probabilistic Quotient Normalization (PQN) $[17,18]$ method and then scaled by the generalized $\log (\lambda)$ transformation $[19,20]$. After NMR post-processing treatment, the spectra were mean-centered and then subjected to unsupervised (principal components analysis; PCA) and supervised (Orthogonal projections to latent structures discriminant analysis; OPLS-DA) multivariate analyses by using SIMCA-P + version 12 (Umetrics, Umea, Sweden).

Metabolites were identified using an in-house NMR database and Chenomx NMR suite v. 7.6 (Chenomx Inc., Alberta, Canada).

\section{Comet assay}

Comet assay was performed as described in Petroni et al. [21]. In detail, microscope slides were firstly precoated with $1 \%$ agarose (Serva Electrophoresis) in PBS without CaMg. Cell suspensions $\left(20 \times 10^{5}\right.$ for each point) were dissociated and resuspended in $10 \mathrm{uL}$ of PBS, then cells were added to $75 \mathrm{uL}$ of low melting agarose $(0.5 \%$ in PBS without $\mathrm{CaMg}$ ): this suspension was cast on the solidified agarose-coated slides and covered by cover slip. After solidification the cover slips were removed again and the slides were immersed in a lysis buffer $(2.5 \mathrm{M} \mathrm{NaCl}$ (Sigma Aldrich), $100 \mathrm{mM}$ EDTA (ethylenediaminetetraacetic acid) (Sigma Aldrich), $10 \mathrm{mM}$ Tris (tris(hydroxymethyl)aminomethane) (Sigma Aldrich), 0.5\% Triton X-100 (SERVA Electrophoresis), $\mathrm{pH}=9.5$ at $4^{\circ} \mathrm{C}$. After the lysis the slides were washed in distilled water then placed in an electrophoretic tank and dipped in cool electrophoresis solution (300 mM NaOH (Sigma Aldrich), $1 \mathrm{mM}$ EDTA (Sigma Aldrich). Electrophoresis was run at $12 \mathrm{~V}$ and $40 \mathrm{~mA}$ for $30 \mathrm{~min}$. After the electrophoresis the slides were dehydrated with ethanol, then rehydrated with $\mathrm{H} 20$. The samples were subsequently stained with ethidium bromide and were visualized at fluorescence microscope (LEICA DM 2500) using tritc filter. Computerized image analysis system (CometScore 1.5) was used to measure several comet parameters (tail length, tail moment, tail \% DNA).

\section{Statistical analysis}

Statistical analysis of experiments was performed using StatView 4.1 software (Abacus Concepts). Statistical differences were analyzed by Student's T-test and a p-value of 0.05 was considered significant. Results are expressed as mean \pm SD from an appropriate number of experiments as indicated in the figure legends.

\section{Results}

\section{NSC exposed to simulated microgravity undergo cell cycle arrest}

NSC derived from 4 days old mouse cerebella [12] were grown under simulated microgravity (SM) for 5 days. After SM cell growth was assessed by cell count, it resulted in $65 \%$ drop in cell proliferation, as shown in Figure 1A. NSC morphology was also investigated: NSC exposed to SM grew as larger and fewer aggregates, as shown in Figure 1B.
We then proceeded to investigate the occurrence of cell division labeling NSC with vital dye AF670. This fluorescent dye binds nonspecifically to cellular proteins; as long as the cells divide the dye is diluted whereas cells that do not divide retain the highest level of fluorescence. After 5 days of SM, 17,5\% of cells were AF670 positive, while in control cells only 4,3\% were still AF670 positive (Figures 1C and 1D), suggesting a difference in the proliferation rate. To gain insight into the mechanism of this phenomenon we analyzed the cell cycle: after SM we observed a drop in G2 phase accompanied by an increase in the S-phase (Figures $1 \mathrm{E}$ and $1 \mathrm{~F}$ ). In line with a block during S-phase, we found that after SM average cell size is increased (supplementary Figure 1A).

To deepen the data obtained from cell cycle analysis we assessed the levels of cyclins in SM compared to $1 \mathrm{G}$ (Figures $1 \mathrm{G}$ and $\mathrm{H}$ ). As shown in Figure 1G, we observed the downregulation of the G2/M progression marker Cyclin B and the G1/S marker Cyclin D2 accompanied by the upregulation of Cyclin A, a marker of S-phase. We also investigated mRNA levels of cyclins and found an apparent discordance in Cyclin B1 and Cyclin D2 levels (Figure 1H). Indeed Cyclin B1 showed a strong transcriptional activation, coherently with cells being in late S-phase, while its protein was downregulated. Thus, we looked for the possible mechanisms involved in such post-transcriptional regulation of cyclins, we found p21 upregulation in SM (supplementary Figure 1B) and speculated that Cyclin B1 protein modulation could be due to $\mathrm{p} 21$ dependent degradation. Moreover, miR-494 and miR-29b-3p, that were shown to target respectively Cyclin B1 and Cyclin D2 and to regulate cell cycle progression $[22,23$ ] were upregulated after SM (supplementary Figure 1C). Altogether these results suggest that cerebellar NSC go through a cell cycle arrest, more specifically in the S-phase.

\section{Metabolic response induced by SM in NSC}

We performed a $1 \mathrm{H}$ NMR-based metabolomic approach to study the effects of simulated microgravity on the metabolism of NSC cells. To this aim, we acquired NMR spectra of cellular extract samples obtained from SM and normal gravity condition experiments at 72 hours.

By means of PCA, we obtained an unsupervised, fully data-driven, overview of the major metabolic differences between cells cultured in the two different conditions. In particular, PC1 and PC2 contributed to the discrimination between microgravity group and control group samples of polar phase, a linear discriminant analysis applied to PC1/ PC2 space demonstrated a net separation in the two groups (Fischer's exact test $\mathrm{p}<0.001$ ), while, the metabolic profiles derived from lipophilic cell extracts provided no discrimination between the two groups. Figures $2 \mathrm{~A}$ and $2 \mathrm{~B}$ NMR show the resulting score plots for both polar and nonpolar metabolite intracellular datasets, respectively.

To determine the best discriminatory metabolites, the NMR profiles of polar intracellular samples were then processed by supervised analysis using OPLS-DA. Supervised analysis minimized the contribution of intergroup variability improving the separation between microgravity and normal gravity groups as shown in Figure 2C NMR. LV1 determined the separation of the two groups, explaining the $33 \%$ of the metabolic variance. Based on the OPLS weight values a series of discriminatory metabolites were identified and are shown in Figure 2D. In particular, this approach indicated that the relative concentrations of the leucine, valine, $\mathrm{N}$-acetyl-groups, methionine, aspartate, asparagine, glutathione, threonine, ATP, phosphocholine and glycerophosphocholine were higher in SM samples compared to normal gravity samples, whereas those of lactate, alanine, homoserine, glutamate, proline, glutamine, glycine, creatine, myo-inositol and phenylalanine were lower. 
A

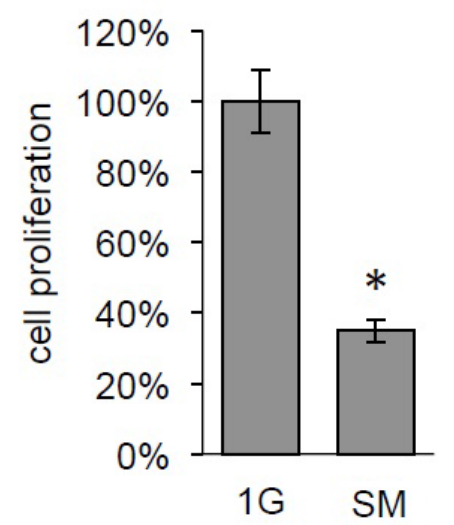

C

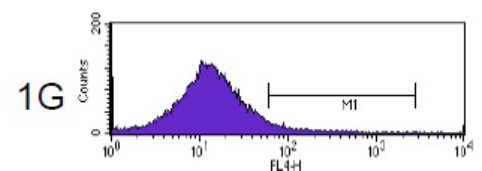

SM

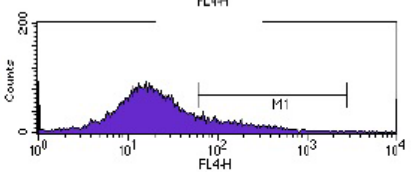

$\mathbf{E}$

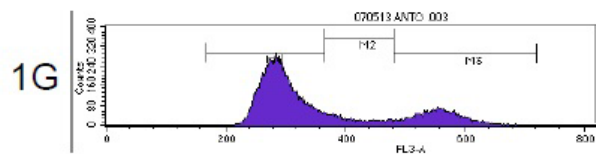

SM

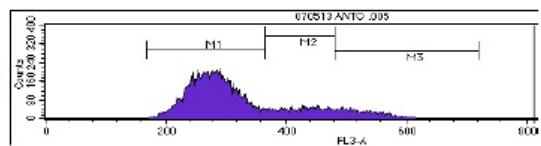

G

$1 \mathrm{G} \quad \mathrm{SM}$

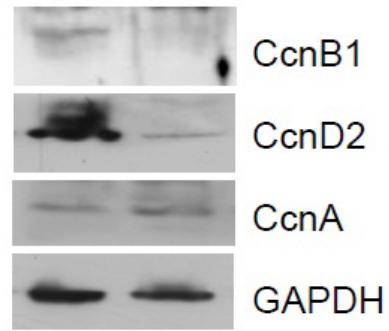

B

$1 \mathrm{G}$

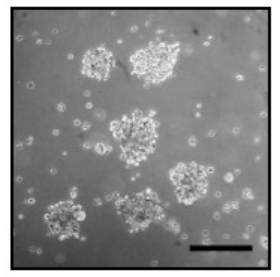

SM

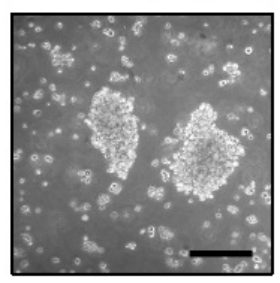

D

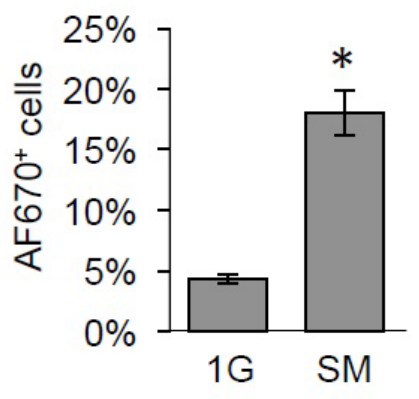

$\mathbf{F}$

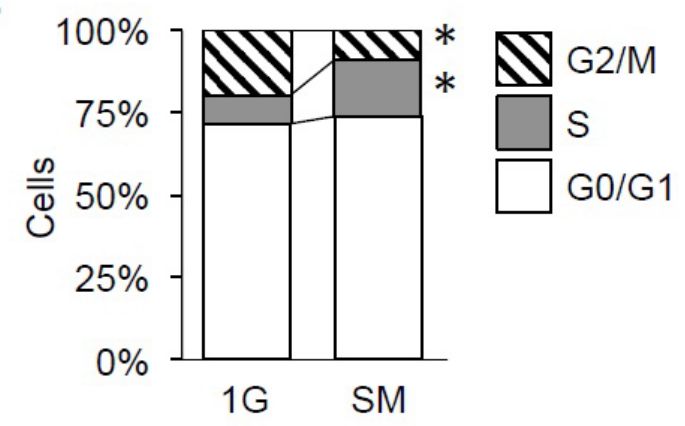

H

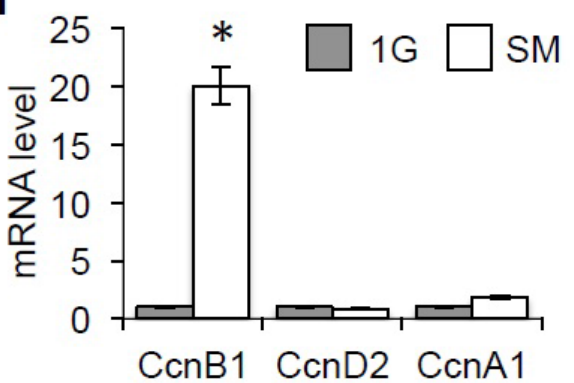

Figure 1: Simulated microgravity induces cell cycle arrest in NSC. (A) Cell proliferation was assessed by cell count after 5 days at simulated microgravity (SM) or normogravity $(1 \mathrm{G})$, where $100 \%$ represent the number of cells in $1 \mathrm{G},{ }^{*} \mathrm{p}<0.05$ (B) Representative images of NSC morphology after 5 days of culture under $1 \mathrm{G}$ or SM. Scale $\mu \mathrm{m}(\mathrm{C})$ bar: Representative image of FACS analysis of Cell Proliferation Dye eFluor ${ }^{\circledR} 670$ marked NSC exposed to SM or $1 \mathrm{G}$ as control. (D) Histogram showing percentage of AF670 positive cells, ${ }^{*}$ p $<0.05$. (E) Representative images of cell cycle profile of NSC grown in SM or $1 \mathrm{G}$. (F) Graph showing the percentage of cells in given cell-cycle phases of NSC grown in SM or $1 G,{ }^{*} p<0.05$. (G) Western blot assay showing cyclins levels together with GAPDH as loading control of NSC grown in $\mathrm{SM}$ or $1 \mathrm{G}$. (H) mRNA levels of cyclins in NSC grown in SM or $1 \mathrm{G},{ }^{*} \mathrm{p}<0.05$. Experiments were performed at least in triplicate. 

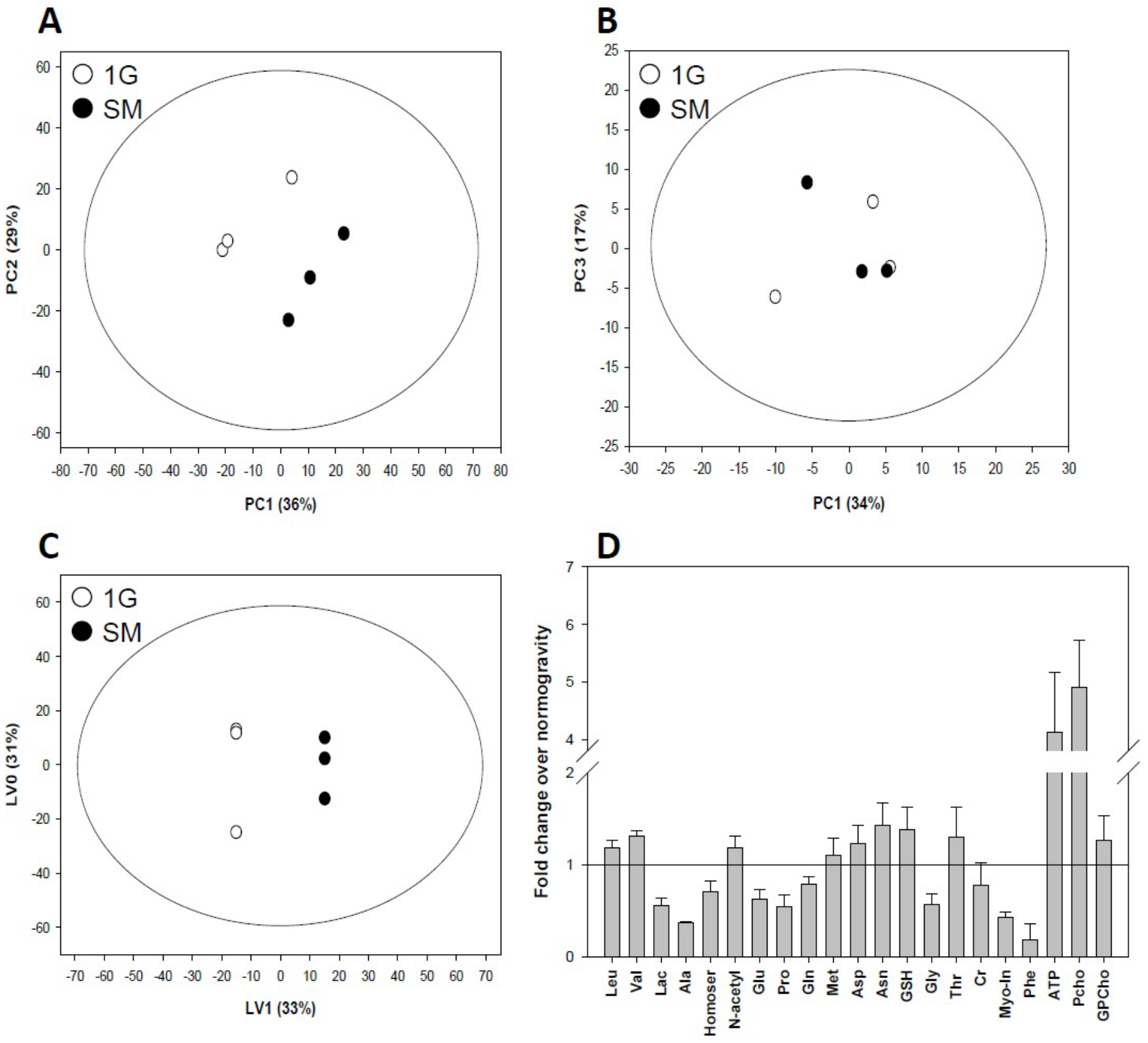

Figure 2: Metabolic analysis of NSC exposed to simulated microgravity. (A-B) Score plots resulting from PCA analyses performed on polar (A) and nonpolar (B) metabolite datasets obtained from SM and normal gravity (1G) condition in three independent experiments. (C) Supervised analysis. (D) Discriminatory metabolites identified in SM vs 1G. Histograms indicate the relative levels of discriminatory metabolites in NSC grown in SM versus $1 \mathrm{G}$ as control. P<0.05 for all metabolites (Leu, leucine; Val, valine; Lac, lactate; Ala, alanine; Homoser, homoserine; N-acetyl, N-acetyl-groups, Glu, glutamate; Pro, proline; Gln, glutamine; Met, methionine; Asp, aspartate; Asn, asparagine; GSH, glutathione; Gly, glycine; Thr, threonine, Cr, creatine; Myo-In, myo-inositol; Phe, phenylalanine; ATP, Adenosine triphosphate; PCho, phosphocholine and GPCho, glycerophosphocholine).

\section{SM is responsible for cellular stress and enhanced apoptosis}

We researched whether changes in proliferation and metabolism in NSC exposed to SM could be accompanied by apoptosis or cellular stress/damage. Indeed we found that SM causes enhanced apoptosis when compared to normogravity, as shown in Figures $3 \mathrm{~A}$ and B by the increase of pyknotic nuclei and in Figure $3 \mathrm{C}$ by the increase of cleaved PARP. Cellular stress was then examined and we found that endogenous HSP70 was strongly upregulated in cells exposed to SM, as shown in Figure 3D. Finally we questioned whether the stress affecting cells exposed to SM could be also associated with DNA damage. Indeed we found that there was a significant upregulation of gamma-H2AX (Figure 3E). Increased DNA lesions, measured by comet assay, were not observed (Figure 3F and G).

\section{NSC in SM transiently lose stemness phenotype}

We then investigated if SM affected stemness features (Figure 4A). We performed clonogenic assay to investigate the content of stem cells in the different culture conditions and we found that after 5 days of 
A

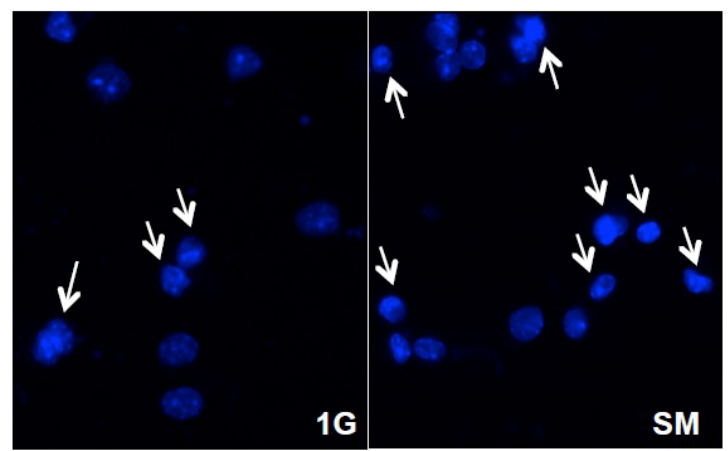

C

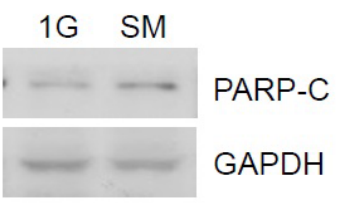

D

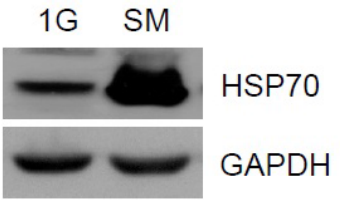

B

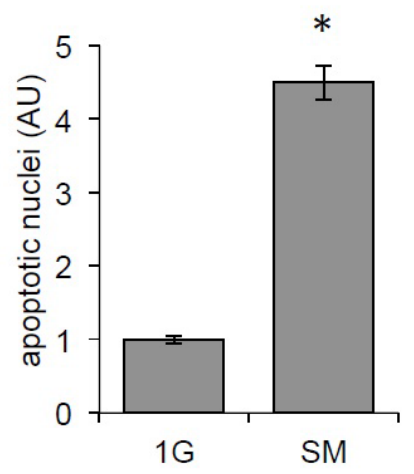

E

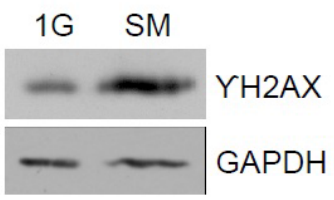

$\mathbf{F}$

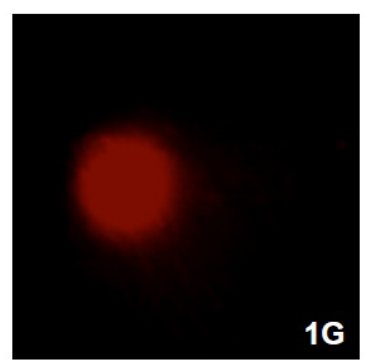

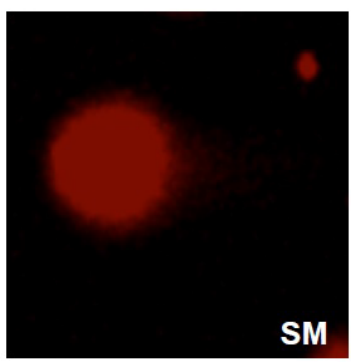

G

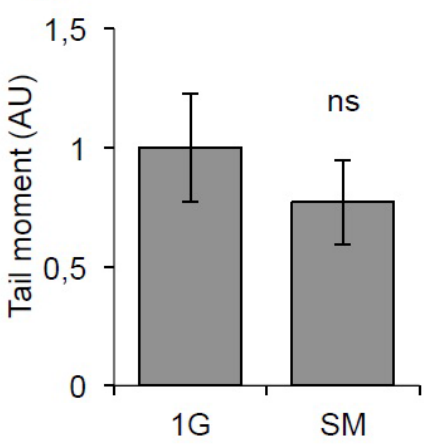

Figure 3: SM causes apoptosis and cellular stress in NSC. (A) Representative images of pyknotic nuclei (indicated by arrows) of cells grown under SM and at $1 \mathrm{G}$ as control stained with DAPI. (B) Histogram showing quantification of manual counts of pyknotic nuclei in NSC grown in SM normalized against $1 \mathrm{G}$ as control; $\mathrm{p}<0.05$ AU: arbitrary units (C) Western blot showing cleaved PARP (PARP-C) levels and GAPDH as loading control in NSC grown in SM and 1G. (D) Western blot showing HSP70 levels and GAPDH as loading control in NSC grown in SM and 1G. (E) Western blot showing active phosphorylated histone H2AX (YH2AX) and GAPDH as loading control in NSC grown in SM and 1G. (F) Representative images of comet assay performed on NSC grown in SM and 1G. (G) Histogram showing normalized tail moment of comet assay performed on NSC grown in SM and $1 \mathrm{G}$, ns: not significant. All experiments were performed in triplicate.

SM clonogenic potential was significantly upregulated (Figure 4B). Furthermore we analyzed the expression of embryonic stem cells marker Nanog [12]. In misleading contrast to the result of clonogenic assay, Nanog expression analysis showed a significant downregulation after 5 days of SM stimulus (Figures $4 \mathrm{C}$ and D). Therefore we assessed whether stemness features could be recovered after few days back in normogravity. Indeed we found that Nanog level returned to its basal expression levels after 5 days of following SM exposure (rescue), as shown in Figures 4C and D.

\section{Post-SM recovery effects on NSC: metabolic features}

To investigate the reversibility of SM effects on the metabolism of NSC, PCA was carried out on a dataset of: a) medium samples obtained from cells cultured in microgravity conditions and collected after 72 hours; b) medium samples obtained from cells recovered from microgravity condition and then cultured in normal condition and collected at 6 and 8 days; c) medium samples obtained from cells cultured in normal gravity condition and collected at 6 and 8 days. Three components were extracted corresponding to $45 \%$ of the total variance. The component space is depicted in Figure 5, which shows the score plot for the first two model components. This plot displays a clear separation among media samples derived from microgravity and normal gravity conditions on PC1. There were no metabolic differences among samples derived from cells of rescue experiments and those derived from cells of normal gravity experiments at 6 and 8 days (t-test $=0.483$ on PC1). 
A

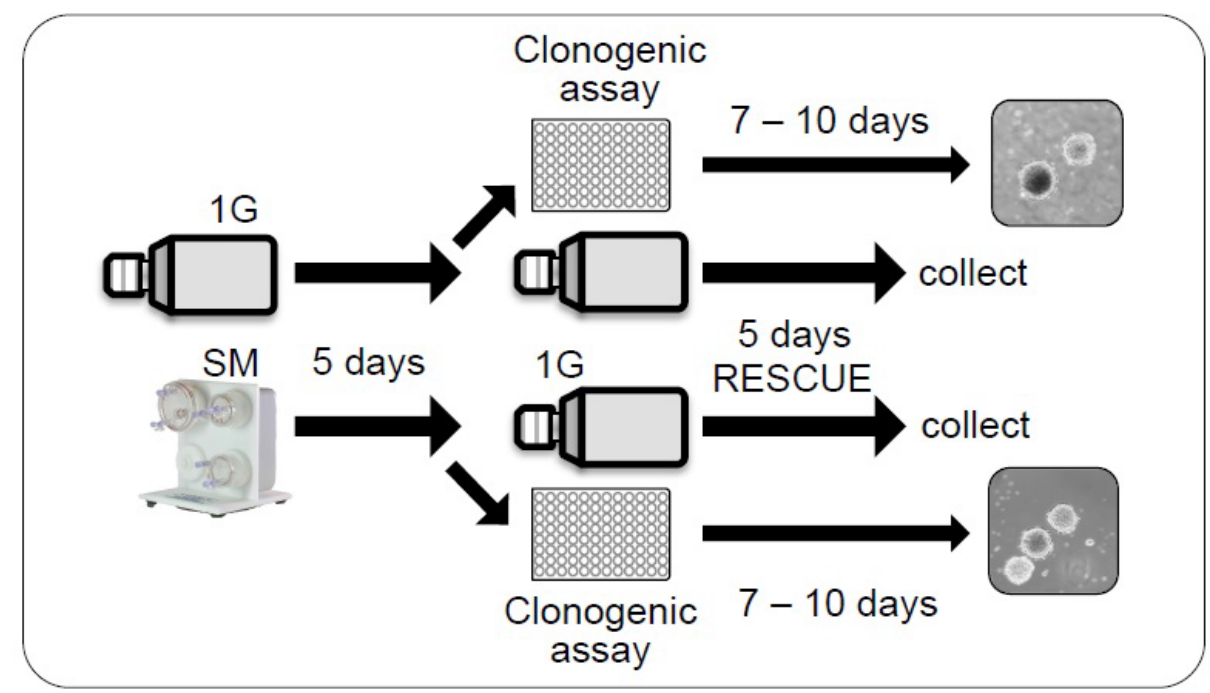

B

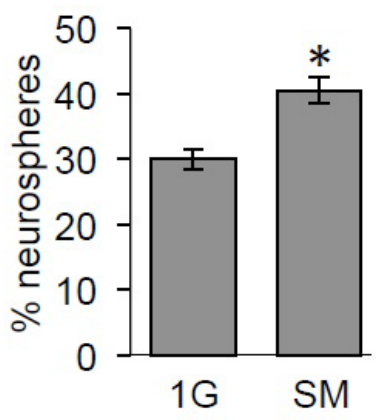

C

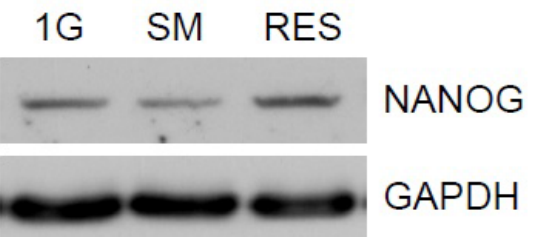

D

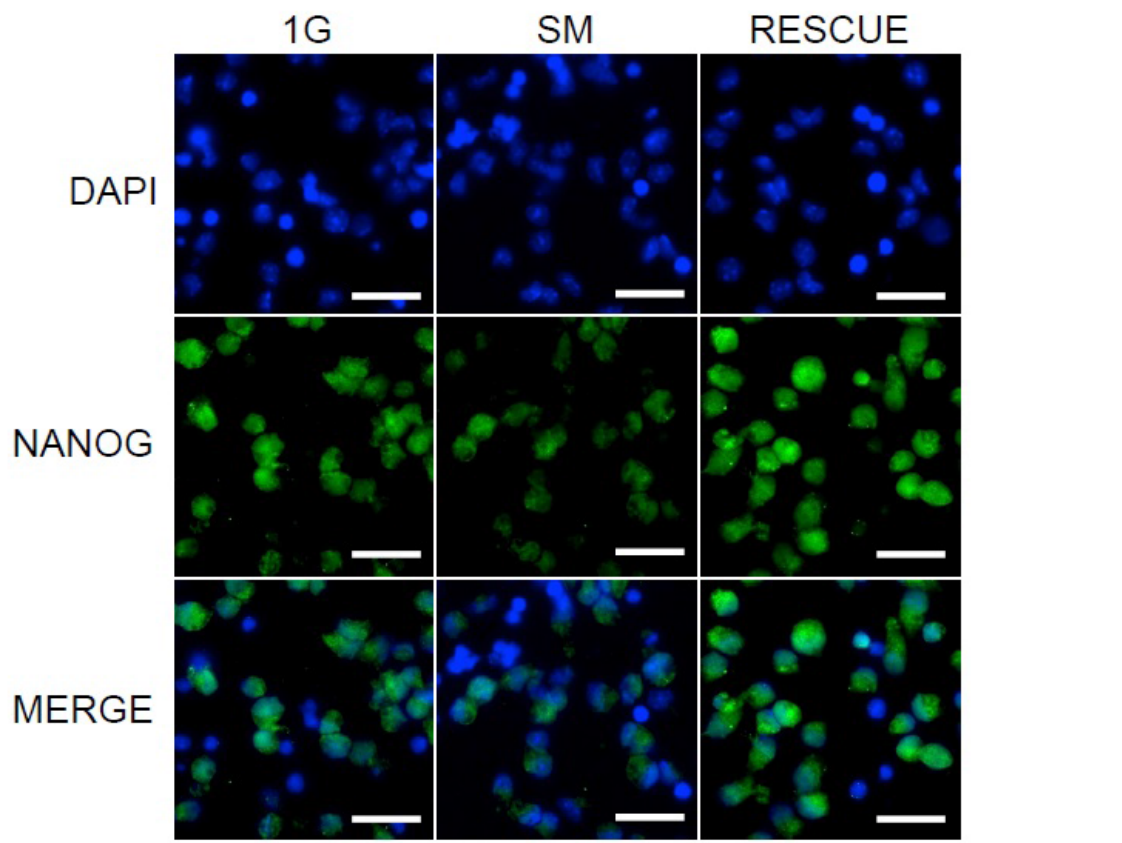

Figure 4: SM transiently affects stemness of NSC. (A) Experimental design for Rescue experiment. After 5 days of SM or $1 \mathrm{G}$ as control, cells were shifted to $1 \mathrm{G}$ for additional 5 days. Cells were also plated for clonogenic assay. (B) Percentage of secondary neurosphere-forming assay of NSC grown in SM and $1 G$ as control, $p<0.05$ (C) Western blot showing Nanog levels and GAPDH as loading control in NSC grown in SM and 1G, RES: rescue. (D) Immunofluorescence staining of Nanog (green) in NSC grown in 1G, SM and after 5 days of recovery in normogravity (RESCUE). Nuclei are counterstained with hoechst (blue); scale bar $10 \mu \mathrm{m}$. Experiments were performed in triplicate. 


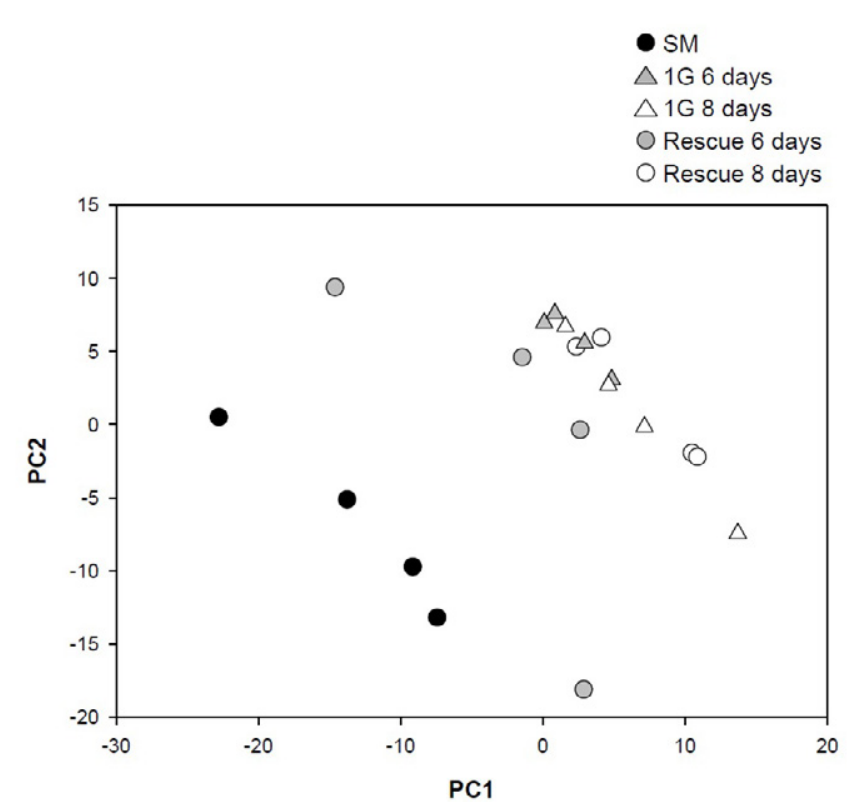

Figure 5: Reversion of metabolic effects induced by microgravity condition in NSC cells as shown by rescue experiment. The score plot of PCA applied to medium samples of three independent rescue experiments shows the absence of metabolic differences among samples derived from media of cells recovered from microgravity condition and then cultured in normal condition and those derived from cells of normal gravity experiments at 6 and 8 days.

\section{Discussion}

Simulated microgravity bioreactors are widely used to investigate the effects of the reduced gravitational force on several biological systems $[2,4,10,24]$. Taking advantage of these techniques, we can get insight in the molecular events underlying the profound changes in the metabolism and the physiology of organisms involved in spaceflights. Researchers involved in space-based biology have struggled in the last 40 years to answer one question: what happens to organisms evolved in gravitational environment when they are forced to live in microgravity? And a subsequent important question is: what happens when they turn back to normal gravity? There is evidence that acclimation to space flight is a complex process involving multiple systems (musculoskeletal, cardiovascular, immunological) [25], but there are no studies on the possible effects of the prolonged but not permanent stay in microgravity on the neural system.

Gravitational modulation was found to have a transient effect on human lymphocyte and lymphoblastoid cells [26] and on human keratinocytes [27], with the conclusion that the time needed for recovery was correlated to the duration of the exposure so SM.

There is experimental evidence that it can take up to 3 years for astronauts to return to the preflight bone and immunological health [25] and that astronauts may be at higher risk for cancer and premature aging $[28,29]$, even if these phenomena were linked to radiation as well as to microgravity. In this paper we research the effects of simulated microgravity on cerebellar NSC. We also investigate the effects of the return to normal gravity after SM, giving a proof of principle of the reversible effects of SM on neural stem cells. There are few papers that explore the effects of microgravity on stem cells: most efforts were conducted in the understanding of the effects of SM on hematopoietic stem cells and muscle and bone precursors [2,4,30,31]; in addition, reports have examined changes that occur in mouse embryonic stem cells [7] and oligodendrocyte precursors [32]. Few reports address the effects of microgravity on stem cells or precursors of the neural lineage. Chiang and colleagues exposed human NSC derived from embryonic stem cells to SM and found that SM would induce cellular proliferation via an increased mitochondrial function [33]. Interestingly also in our experiments ATP content is increased when compared to normogravity. We could speculate that cells respond to the reduced gravity with an initial attempt to higher proliferation followed by a cellcycle progression arrest.

Indeed, our experimental results indicate that exposing NSC to SM induces inhibition of cell proliferation. This is in line with what has been reported in other contexts, such as mesenchymal and embryonic stem cells $[7,34]$. More specifically, cerebellar NSC appears to be stuck in S-phase and not progressing in cell cycle. Previous works have shown that simulated microgravity can induce the rearrangement, or even depolymerization of cytoskeleton of human cells [35]. However, the role of cytoskeleton in cell modifications in SM and its possible consequences on cell proliferation is still an open question.

We show that SM induced apoptosis in agreement with several studies demonstrating the frequent association between cell cycle arrest and cell death [36,37].

Interestingly, we find that HSP70 protein, a chaperone involved in the refolding, is strongly modulated by microgravity in NSC. HSP70 has an important role in cancer stem cells from Medulloblastoma [38] that share several molecular mechanisms with cerebellar NSC [12]. This result confirms what has been already published in endothelial cells cultured in SM that sense microgravity as a stressful condition and over express HSP70 to maintain their proliferative potential [39].

We also show that NSC expresses higher levels of a marker of DNA damage; nevertheless, this data is not associated with evident breaks in DNA, as suggested by the unchanged comet assay. H2AX is a critical factor of the S/G2 DNA-damage checkpoint complex [40]. It has been reported that $\mathrm{H} 2 \mathrm{AX}$ phosphorylation blocks DNA synthesis and cell cycle progression in NSC [41,42]. Moreover NSC self-renewal and NSC niche size can be dynamically modulated by targeting H2AX activation through GABA R signaling [41]. Accordingly, we speculate that in our NSC grown in SM, H2AX phosphorylation could be linked to effects of SM on S-phase block. However, little is known about the effect of microgravity on cellular DNA damage. We reveal no overt DNA damage in NSC after SM by DNA tail comet assays. Degan et al. reported that exposure of freshly drawn lymphocytes and lymphoblastoid cells to SM for 24 or 72 hours is not significantly associated with the induction of DNA damage [26]. However, Kumari et al. reported that the exposure of human lymphocytes to SM for 7 days significantly increased the level of DNA damage [6]. Therefore prolonged exposure to SM can induce DNA damage while short pulse of SM culture can lead to a stressful process. NSC appears to be extremely sensitive to SM-induced stress as suggested by the higher levels of stress protein and apoptosis rate detected in comparison to normogravity. Our results show that main effects of SM on NSC reside in the cell cycle arrest, induced apoptosis and the activation of replicative stress response through high levels of phosphorylated H2AX without affecting cell genomic stability.

Interestingly, when shifted back to normal gravity, NSC showed enhanced clonogenic ability despite the downregulation of stemness marker Nanog. We might suppose that cells previously blocked in their cell cycle progression, find in the normal gravity the possibility to complete their cell cycle, leading to increased clonogenicity. 
Metabolomics has recently emerged as a non-targeted approach for identifying metabolic changes caused by external stimuli $[14,43]$. To our knowledge, this is the first report of a metabolic approach applied to SM studies. Interestingly, we determine metabolites that are modulated by SM. Moreover we found by metabolic footprint that the effects of SM on NSC are transient, coherently with our results on stemness features. Moreover no permanent effect of SM is detected: the block in S-phase is overcome when cells return to normogravity and, more importantly, NSC clonogenic ability is not impaired. On the contrary, we even observed increased clonogenic ability shortly after SM exposure. We speculate that this phenomenon may be due to the recovery of NSC cell cycle progression. Indeed, cells are released from the block in S-phase and are able to complete cell divisions.

\section{Conclusion}

Taken together, our data suggest that SM has a deep effect on NSC that involves changes in cell cycle and apoptosis, likely due to events that involve gravity-sensitive molecules in the cytoskeleton. However, these events have only temporary negative effects on NSC, since their stemness properties are not impaired. Effects of SM on NSC are transient, since after few days in normogravity, both stemness marker Nanog and metabolic features return to basal levels.

\section{Acknowledgments}

Silvano M, Miele $\mathrm{E}$ and Valerio MC equally contributed to this work. Ferretti $\mathrm{E}$ and $\mathrm{Po} A$ are co-senior author. This work was supported by Italian Space Agency (ASI), FIRB, PRIN, PON01 \#01059 and PON01 \#02464, Fondazione Cenci Bolognetti and Italian Institute of Technology (IIT) projects.

\section{References}

1. Graebe A, Schuck EL, Lensing P, Putcha L, Derendorf H (2004) Physiological, Pharmacokinetic, and Pharmacodynamic Changes in Space. J Clin Pharmacol 44: 837-853. [PubMed]

2. Damm Benavides T, Richard S, Tanner S, Wyss F, Egli M, et al. (2013) Calcium-dependent deceleration of the cell cycle in muscle cells by simulated microgravity. FASEB J 27: 2045-2054. [PubMed]

3. Nabavi N, Khandani A, Camirand A, Harrison R (2011) Effects of microgravity on osteoclast bone resorption and osteoblast cytoskeletal organization and adhesion. Bone 49: 965-974. [PubMed]

4. Plett P, Abonour R, Frankovitz S, Orschell C (2004) Impact of modeled microgravity on migration, differentiation, and cell cycle control of primitive human hematopoietic progenitor cells. Exp Hematol 32: 773-781. [PubMed]

5. Damm Benavides T, Franco-Obregón A, Egli M (2013) Gravitational force modulates $\mathrm{G} 2 / \mathrm{M}$ phase exit in mechanically unloaded myoblasts. Cell Cycle 12: 3001-3012. [PubMed]

6. Kumari R, Singh K, Dumond J Jr (2009) Simulated microgravity decreases DNA repair capacity and induce DNA damage in human lymphocytes. J Cell Biochem 107: 723-731. [PubMed]

7. Wang $Y$, An L, Jiang $Y$, Hang $H$ (2011) Effects of simulated microgravity on embryonic stem cells. PLoS One 6: e29214. [PubMed]

8. Yan M, Wang Y, Yang M, Liu Y, Qu B, et al. (2015) The effects and mechanisms of clinorotation on proliferation and differentiation in bone marrow mesenchymal stem cells. Biochem Biophys Res Commun 460: 327-332. [PubMed]

9. Girardi C, De Pittà C, Casara S, Calura E, Romualdi C, et al. (2014) Integration analysis of microRNA and mRNA expression profiles in human peripheral blood lymphocytes cultured in modeled microgravity. Biomed Res Int 2014: 296747. [PubMed]

10. Singh K, Kumari R, Dumond J (2010) Simulated microgravity-induced epigenetic changes in human lymphocytes. J Cell Biochem 111: 123-129. [PubMed]

11. Wei L, Liu C, Kang L, Liu Y, Shi S, et al. (2014) Experimental study on effect of simulated microgravity on structural chromosome instability of human peripheral blood lymphocytes. PLoS One 9: e100595. [PubMed]

12. Po A, Ferretti E, Miele E, De Smaele E, Paganelli A, et al. (2010) Hedgehog controls neural stem cells through p53-independent regulation of Nanog. EMBO J 29: 2646-2658. [PubMed]
13. Garg N, Po A, Miele E, Campese A, Begalli F, et al. (2013) microRNA-17-92 cluster is a direct Nanog target and controls neural stem cell through Trp53inp1. EMBO J 32: 2819-2832. [PubMed]

14. Priori R, Scrivo R, Brandt J, Valerio M, Casadei L, et al. (2013) Metabolomics in rheumatic diseases: the potential of an emerging methodology for improved patient diagnosis, prognosis, and treatment efficacy. Autoimmun Rev 12: $1022-$ 1030. [PubMed]

15. Bligh E, Dyer W (1959) A rapid method of total lipid extraction and purification Can J Biochem Physiol 37: 911-917. [PubMed]

16. Tiziani S, Emwas A, Lodi A, Ludwig C, Bunce C, et al. (2008) Optimized metabolite extraction from blood serum for $1 \mathrm{H}$ nuclear magnetic resonance spectroscopy. Anal Biochem 377: 16-23. [PubMed]

17. Dieterle F, Ross A, Schlotterbeck G, Senn H (2006) Probabilistic quotient normalization as robust method to account for dilution of complex biological mixtures. Application in 1H NMR metabonomics. Anal Chem 78: 4281-4290. [PubMed]

18. Dieterle F, Riefke B, Schlotterbeck G, Ross A, Senn H, et al. (2011) NMR and MS methods for metabonomics. Methods Mol Biol 691: 385-415. [PubMed]

19. Parsons H, Ludwig C, Günther U, Viant M (2007) Improved classification accuracy in 1- and 2-dimensional NMR metabolomics data using the variance stabilising generalised logarithm transformation. BMC Bioinformatics 2: 234. [PubMed]

20. Purohit $P$, Rocke D, Viant M, Woodruff D (2004) Discrimination models using variance-stabilizing transformation of metabolomic NMR data. OMICS 8: 118130. [PubMed]

21. Petroni M, Sardina F, Heil C, Sahun-Roncero M, Colicchia V, et al. (2015) The MRN complex is transcriptionally regulated by MYCN during neural cell proliferation to control replication stress. Cell Death Differ. [PubMed]

22. Yamanaka S, Campbell N, An F, Kuo S, Potter J, et al. (2012) Coordinated effects of microRNA-494 induce $\mathrm{G} \square / \mathrm{M}$ arrest in human cholangiocarcinoma Cell Cycle 11: 2729-2738. [PubMed]

23. Gong J, Li J, Wang Y, Liu C, Jia H, et al. (2014) Characterization of microRNA-29 family expression and investigation of their mechanistic roles in gastric cancer Carcinogenesis 35: 497-506. [PubMed]

24. Blaber E, Dvorochkin N, Torres M, Yousuf R, Burns B, et al. (2014) Mechanical unloading of bone in microgravity reduces mesenchymal and hematopoietic stem cell-mediated tissue regeneration. Stem Cell Res 13: 181-201. [PubMed]

25. Williams D, Kuipers A, Mukai C, Thirsk R (2009) Acclimation during space flight: effects on human physiology. CMAJ 180: 1317-1323. [PubMed]

26. Degan P, Sancandi M, Zunino A, Ottaggio L, Viaggi S, et al. (2005) Exposure of human lymphocytes and lymphoblastoid cells to simulated microgravity strongly affects energy metabolism and DNA repair. J Cell Biochem 94: 460469. [PubMed]

27. Clement J, Lacy S, Wilson B (2008) Gene expression profiling of human epidermal keratinocytes in simulated microgravity and recovery cultures. Genomics Proteomics Bioinformatics 6: 8-28. [PubMed]

28. Hamm P, Billica R, Johnson G, Wear M, Pool S (1998) Risk of cancer mortality among the Longitudinal Study of Astronaut Health (LSAH) participants. Aviat Space Environ Med 69: 142-144. [PubMed]

29. JD S (2013) Spaceflight-induced bone loss: is there an osteoporosis risk? Curr Osteoporos Rep 11: 92-98. [PubMed]

30. Tamma R, Colaianni G, Camerino C, Di Benedetto A, Greco G, et al. (2009) Microgravity during spaceflight directly affects in vitro osteoclastogenesis and bone resorption. FASEB J 23: 2549-2554. [PubMed]

31. Zheng L, Liu J, Hu Y, Zhong T, Xiong S, et al. (2011) Simulated microgravity erythroid differentiation, and the expression of transcription factor GATA-1 in CD34+ cells. Aviat Space Environ Med 82: 513-517. [PubMed]

32. Espinosa-Jeffrey A, Paez P, Cheli V, Spreuer V, Wanner I, et al. (2013) Impact of simulated microgravity on oligodendrocyte development: implications for central nervous system repair. PLoS One 8: e76963. [PubMed]

33. Chiang $M$, Lin $H$, Cheng $Y$, Yen $C$, Huang $R$, et al. (2012) $\beta$-adrenocepto pathway enhances mitochondrial function in human neural stem cells via rotary cell culture system. J Neurosci Methods 207: 130-136. [PubMed]

34. Dai Z, Wang R, Ling S, Wan Y, Li Y (2007) Simulated microgravity inhibits the 
Citation: Silvano M, Miele E, Valerio MC, Casadei L, Begalli F et al. (2015) Consequences of Simulated Microgravity in Neural Stem Cells: Biological Effects and Metabolic Response. J Stem Cell Res Ther 5: 289. doi:10.4172/2157-7633.1000289

proliferation and osteogenesis of rat bone marrow mesenchymal stem cells. Cell Prolif 40: 671-684. [PubMed]

35. Buravkova L, Romanov Y (2001) The role of cytoskeleton in cell changes under condition of simulated microgravity. Acta Astronaut 48: 647-650. [PubMed]

36. Lossi L, Mioletti S, Merighi A (2002) Synapse-independent and synapsedependent apoptosis of cerebellar granule cells in postnatal rabbits occur at two subsequent but partly overlapping developmental stages. Neuroscience 112: 509-523. [PubMed]

37. Rogakou E, Nieves-Neira W, Boon C, Pommier Y, Bonner W (2000) Initiation of DNA fragmentation during apoptosis induces phosphorylation of $\mathrm{H} 2 \mathrm{AX}$ histone at serine 139. J Biol Chem 275: 9390-9395. [PubMed]

38. Ronci M, Catanzaro G, Pieroni L, Po A, Besharat ZM, et al. (2015) Proteomic analysis of human sonic hedgehog $(\mathrm{SHH})$ medulloblastoma stem-like cells. Mol Biosyst 11: 1603-1611. [PubMed]
39. Cotrupi S, Maier J (2004) Is HSP70 upregulation crucial for cellular proliferative response in simulated microgravity? J Gravit Physiol 11: 173176. [PubMed]

40. Fernandez-Capetillo O, Lee A, Nussenzweig M, Nussenzweig A (2004) H2AX: the histone guardian of the genome. DNA Repair (Amst) 3: 959-967. [PubMed]

41. Andäng $M$, Hjerling-Leffler J, Moliner A, Lundgren T, Castelo-Branco G, et al (2008) Histone H2AX-dependent GABA(A) receptor regulation of stem cell proliferation. Nature 451: 460-464. [PubMed]

42. Fernando R, Eleuteri B, Abdelhady S, Nussenzweig A, Andäng M, et al. (2011) Cell cycle restriction by histone $\mathrm{H} 2 \mathrm{AX}$ limits proliferation of adult neural stem cells. Proc Natl Acad Sci USA 108: 5837-5842. [PubMed]

43. Blandino G, Valerio M, Cioce M, Mori F, Casadei L, et al. (2012) Metformin elicits anticancer effects through the sequential modulation of DICER and c-MYC. Nat Commun 3: 865. [PubMed] 